\title{
Ayurvedic Antivirals for Management of COVID-19 Patients
}

\author{
Kadamb Patel ${ }^{1}$, Bharati Patel ${ }^{2}$ and Shabbir Moochhala ${ }^{2,3 *}$ \\ ${ }^{1}$ Centre for Molecular Diagnostics, School of Applied Science, Temasek Polytechnic, Singapore \\ ${ }^{2}$ Department of Surgery, Yong Loo Lin School of Medicine, National University of Singapore, Singapore \\ ${ }^{3}$ Department of Pharmacology, Yong Loo Lin School of Medicine, National University of Singapore, Singapore \\ *Corresponding author: Shabbir Moochhala, Department of Pharmacology, Yong Loo Lin School of Medicine, National University \\ of Singapore, Singapore
}

ARTICLE INFO

Received: 慧 June 10, 2020

Published: 幽 June 19, 2020

Citation: Kadamb P, Bharati P, Shabbir M. Ayurvedic Antivirals for Management of COVID-19 Patients. Biomed J Sci \& Tech Res 28(3)-2020. BJSTR. MS.ID.004643.

Abbreviations: TMs: Traditional medicines; TKM: Traditional Korean medicine; TCM: Traditional Chinese medicine; SARSCoV-2: Severe acute respiratory syndrome coronavirus 2; MERS-CoV Middle East Respiratory Syndrome corona virus; WHO: World health organization
ABSTRACT

COVID-19 pandemic has drawn urgent global attention. Antiviral drugs are available for treating symptoms of respiratory viral infections; currently, there is no preventive or treatment medicine available. Effective medicine is limited and not reaching masses. In the absence of a vaccine, the situation is stretched in developing nations. Even if the vaccine is developed there are other correlated issues like the emergence of mutant and more virulent strains of the virus, In view of this, complementary and alternative medicine offers plenty of preventive and treatment options which can be implemented for masses in a short period. Polyherbal formulations using medicinal herbs with a wide variety of properties like antiviral, anti-inflammatory, antioxidant and immunomodulating can be effectively deployed for management of current COVID-19 pandemic. Potential clinically tested medicinal herbs with antiviral properties are presented with its possible mode of action. Currently, these medicinal herbs are actively used, either singly or in combination, for the preventive or treatment of COVID-19 or respiratory viral infections.

Keywords: COVID-19; Medicinal herbs; Traditional medicine; Traditional Chinese medicine; Ayurveda; Kampo; Traditional Korean medicine; Unani; Antiviral; Antiinflammatory; Antioxidant; Immunomodulator

\section{Introduction}

The world is battling a "very intense outbreak" of the coronavirus infection (COVID19), and its economic impact on the world is more severe than that from SARS in 2003. Coronaviruses are enveloped viruses with positive single-stranded RNA genome [1]. So far, humans are infected with six known strains of coronaviruses. Recently a novel coronavirus (COVID-19) was detected in Wuhan, China $[2,3]$. Similar to highly pathogenic coronaviruses SARS-CoV and MERS-CoV, COVID-19 also caused severe respiratory diseases. COVID-19 was started by zoonotic transmission likely from bats and spread rapidly among humans [4]. At present, there are potential drugs which are successful in eradicating SARS-CoV. Currently, remdesivir is a most promising antiviral drug for COVID-19, which is under clinical development for the treatment of Ebola virus infection [5].
The coronavirus may take longer to be eradicated here compared to the severe acute respiratory syndrome, or SARS, with confirmed cases of COVID-19 globally exceeding one million-plus and more countries going into lockdown to slow the widespread COVID-19 infection. There is a general agreement that the prevalent COVID-19 infection will decline with the development of herd immunity. That transpires when a large number of people in a community develop immunity against a pathogen. There are two outcomes: One is immunization. Researchers must develop a safe and effective vaccine against the coronavirus, and health authorities would have to get it to enough people. The second path to herd immunity is grimmer: It can also come about after a large portion of a community is infected with a pathogen and develops resistance to it that way. 
The COVID-19 presents several uncertainties, and none of us can eliminate our risk of getting infected with COVID-19. The best option is to build up immunity against diseases with healthy food and sound sleep. The robust immune system is vital for fighting COVID -19 infection. Recent studies show improving nutrition helps enhance immune function. Significant evidence suggests that the immune system declines with age; thus, there will be less immune response upon infectious challenge $[2,3,6]$. Many reports are quick to attribute immune senescence to adaptive immunity dysfunction $[4,7]$ while dismissing the possibility of altered innate immunity. Others indicate the contrary, demonstrating that innate cells are also affected in advanced age [8,9]. In the absence of a vaccine, the best option is to improve immunity against COVID-19 by various means like health supplements, medicine, healthy nutritious food, and active lifestyle. Nutraceuticals or holistic health supplements which can enhance immunity against viruses and bacteria are today's, and future market needs Researchers at the Peter Doherty Institute for Infection and Immunity in Australia, examined blood samples from a healthy 47-year-old woman. She contracted COVID-19 and found that her recovery was due to an unexpectedly strong immune response. A scientific article on the case was published in Nature Medicine [10].

In summary, the paper says the robust immune system does help in recovering from COVID-19 infection. Another recent most studies on COVID-19 by Neil Ferguson and his colleagues from Imperial College London have been trying to figure out the key reasons for older people's greater susceptibility to the virus and, in particular, why some mount a more robust immune response than others. Research paper summarizes ageing, and declining immunity is responsible for COVID-19 infection outcome [11]. Ageing and declining immunity could be a significant problem associated with COVID-19 infections. Recent data on COVID-19 from China showed that comorbidities dramatically raise the risk of dying from COVID-19. Chronic illnesses maybe not only a contributor to COVID-19 deaths, biological ageing, and declining immunity was also responsible for increased deaths among COVID-19 patients [12]. In the prevention and treatment of COVID-19, Traditional Chinese medicines have received broad acceptance, especially in treating cases with mild symptoms [13]. Iwu et al. reported on the use of simple medicinal plant crudes for therapeutic purposes, known as first-generation plant drugs [14]. Several effective medicines were used in their natural state as therapeutic agents by populations from all over the world, based on the empirical studies of their application. These medicinal plant extracts became vital constituents in treating severe diseases caused by several pathogens [15].

Infectious diseases account for half of all diseases in developing countries, and according to the WHO, 80\% of world populations depend on traditional medicines (plant extracts) for their treatment [16]. Estimated Twenty-five per cent of drugs currently in use is plant derived. Over time, massive flora from different environments has set new foundations for plant-derived complexes with therapeutic applications as antiviral agents [17]. Natural products of some plants contain a variety of metabolites. A vital feature of some of these metabolites is that they impede virus replication without affecting the metabolism of the host, making them ideal candidates for the development of useful antiviral drugs with limited side effects. In addition to their ability to interfere directly with viral replication, these natural plant products may also lead to the modification or enhancement of the host immune response against viruses; hence, alleviating the symptoms and reducing mortality caused by viral infections [18]. Keeping in view the global burden of diseases caused by viruses, there is an urgent need to develop safe and effective antiviral drugs. Currently, medicinal plants and their bioactive metabolites are the focus of interest, which may provide desirable treatment strategies for the people of developing countries where the majority of the population cannot afford expensive western medicine [19].

\section{Medicinal herbs and Traditional wisdom}

Traditional medicines (TMs) use natural herbs. Traditional Chinese Medicine (TCM), Traditional Korean Medicine (TKM), Ayurveda, Kampo and Unani they all employ natural herbal products. They have been practised universally for centuries, and they have emerged as alternative medicine. They may have certain flaws, but they are still a valuable repository of human knowledge [20]. Traditional medicine is used since ages its the oldest form of health care system in the world and is commonly used for preventive, and treatment of physical and mental diseases. Various civilizations developed a functional healing process to overcome a variety of health and critical diseases. TM is also known as complementary and alternative, or ethnic medicine; it still plays a crucial role in many countries today for the prevention and management of a wide array of diseases [21]. Kampo is the TM of Japan. TCM was introduced to Japan from China; TCM has been significantly altered and adapted by Japanese practitioners to meet their needs and gradually evolved into Kampo. A recent study has found that several physicians in Japan are using traditional Kampo medicines as preferred medication in their daily practice $[22,23]$.

Unani originated from ancient Greek civilization; it is a holistic medical system with a history that can be traced back to 2500 years [24]. Unani has attracted significant attention all over the world, especially in India, where it has been integrated into the national health care system [25]. Ayurveda is the traditional Indian system of medicine, has been extensively used since ages and serval ayurvedic herbs from the Indian subcontinent have been used for preventing and curing human disease [26]. Since ancient times, Indian herbs have been used as a treatment and preventive strategy for several diseases, including respiratory viral infections. Herbs are used in viral respiratory infections to build a robust immune system. The universal approach of AYUSH systems of medicine focuses on prevention through prophylactic interventions, dietary 
management, and lifestyle modification for improving the immunity [27]. Following are the key clinically tested antiviral Ayurvedic medicinal herbs which may be beneficial for the treatment of COVID 19

a. Glycyrrhiza glabra. Glycyrrhiza glabra contains anethole ("trans"-1- methoxy-4-(prop-1-enyl)benzene) and polysaccharides (glycyrrhizin) [28]. The other active compounds of glycyrrhiza glabra include Saponins, Triterpine, and Glycyrrhizic acid these compounds have the potential ability to inhibits respiratory virus replication [29]. Glycrrhizic acid stimulates macrophages [30] and hence elevate and assist immune stimulation [31]. In vitro study suggests that glycyrrhizin inhibits viral adsorption and penetration hence showcase anti-SARS-CoV effects [32]. In randomized phase II trials, patients with chronic hepatitis reported glycyrrhizin has shown to reduce transaminase levels [33]. Several studies have demonstrated that glycyrrhizin showed a significant inhibiting effect on influenza virus [34]. In Japan, Glycyrrhiza glabra has been used for more than two decades a treatment for chronic hepatitis [35]. Studies have reported that glycyrrhizin has an antiviral effect on coronaviruses, which leads to a possibility to explore glycyrrhizin as an experimental drug for the COVID-19 therapy or it can lead to the development of more effective drugs [36].

b. Recently Chen et al. reported the host receptor for COVID-19 Angiotensin-converting enzyme 2 (ACE2), is the same as the host receptor for SARS-CoV, which opens up new possibilities to block Angiotensin-converting enzyme 2 for preventing and inhibiting COVID-19 infection. Molecular docking studies using Angiotensin-converting enzyme 2 predict their capacity to prevent COVID-19 infection [37]; thus, it has been proposed that glycyrrhizin is worth further investigation for preventing COVID-19 infection

c. Ocimum sanctum. O. sanctum, also known as Holy Basil. The primary chemical constituents of 0 . sanctum are $\beta$-caryophyllene Rosmarinic acid, Ursolic acid, Linalool, Eugenol, Carvacrol and Oleanolic acid [38]. In last decades Ocimum sanctum is extensively investigated, one of the active component eugenol has a wide variety of biological activities such as antibacterial activity [39], antifungal activity [40] and antiviral activity [41].

d. Ghoke (2018) reported that crude extract Ocimum could be a promising extract for developing safe and efficacious antiviral compound against the H9N2 virus. The protective efficacy of Ocimum is credited to serval mechanisms of action, for example, specific inhibition of a stage in viral intracellular multiplication and non-specific interference with virus-cell interactions like masking/blocking the HA glycoprotein [42]

e. Allium sativum, also known as Garlic, belongs to family Alliaceae. Garlic is a rich source of flavanols like Isorhamnetin,
Kaempferol Myricetin, and Quercetin Flavonoids present in Allium sativum have a strong inhibitory effect on virus multiplication it's also blocks the synthesis of proteins and genetic material in the virus [43-45]. The effectiveness of quercetin is associated with the ability of this chemical to target cellular processes during virus infection. Studies have reported that quercetin plays a crucial role in preventing viral entry or blocking proteins required for viral replication. Quercetin is known to be effective against viruses like influenza type A virus (IAV), Hepatitis viruses and Poliovirus [43].Quercetin blocks the entry of the virus in the host cells by inhibiting adhesion of viral glycoproteins to host cells [46].

f. Wu et al. (2016) reported the role of quercetin in blocking the entry of the virus to host cell by interacting with Haemagglutinin protein, which leads to inhibition of membrane fusion [47]. Similarly, Yao et al. reported the role of quercetin in blocking viral attachment in enterovirus infections [48]. In another study, Quercetin 3-0-D-glucoside, a derivative of quercetin, has inhibited the entry of Ebola virus in the host cell [49]. Allium sativum thus could be proposed as a potent antiviral candidate

g. Cocos nucifera. It has been reported that medium-chain fatty acids (MCFA), and their derivatives (e.g., monoglycerides) of Cocos nucifera oil have potent antiviral properties [50]. The antiviral action is credited to monolaurin (Lauric acid monoglyceride), which has the unique ability to solubilize the virus outer membrane envelope phospholipids, which causes the disintegration of the virus. There are growing pieces of evidence which suggest that MCFA obstructs signal transduction process of an organism [51], which inhibits virus envelop assembly and virion maturation [52].

h. Azadirachta indica, commonly known as neem tree. Three bitter compounds which have medicinal values are extracted from neem oil are nimbinin, nimbin, and nimbidin, respectively [53]. The seeds contain complex secondary metabolite azadirachtin all parts of the plant yield $\beta$-sitosterol. The Azadirachtin, Nimbin, and Nimbidin have potential antiviral properties [54]; the exact mode of action is based on interference in viral production. Azadirachta indica leaf extract pure compound (Azadirachtin) has shown encouraging results in inhibiting replication of Dengue virus type 2 . Thus, neem can be a potential source of antiviral medicine [55].

i. Strobilanthes cusia. Strobilanthes cusia belongs to Acanthaceae family, is widely distributed in Taiwan, Myanmar, the Himalayan region, India, Bangladesh and China. Strobilanthes root and leaf extracts have potential antimicrobial, antipyretic, antiviral and anti-inflammatory activities [56]. The Strobilanthes roots are used for the treatment of common respiratory virus infections, including coronavirus [57]. The methanol extract of Strobilanthes roots 
and leaf contains indigodole $\mathrm{A}$, and indigodole B, tryptanthrin, indirubin, betulin and $\beta$-sitosterol [58]

j. Several bioactive components from the Strobilanthes roots like, 3H-enzoxazolinone, and urantiamide acetate, trobilanthes A have exhibited antiviral activity against respiratory viruses' infections [59]. Strobilanthes leaves contains chemical components like indigodole $\mathrm{A}$, indigodole B (5aR-ethyltryptanthrin), indirubin, $\beta$-sitosterol, betulin, tryptanthrin (6,12-dihydro-6,12-dioxoindolo-(2,1-b)quinazoline),strobilanthosides $\mathrm{A}-\mathrm{C}$, and phenylethanoid glycosides. These chemical components possess antitumor, anti-inflammatory, and antibacterial activities [60]. Specifically, indirubin and its derivatives have been suggested as an immunomodulatory and antiviral agent against Influenza A virus infection due to their ability to regulate Influenza A virus-induced inflammation [61]. Recently Tsai et al. reported a potential antiviral mechanism where Strobilanthes cusia blocked the viral RNA genome synthesis and induced papainlike protease activity targeting the $\mathrm{HCoV}$ [57].

k. Camellia sinensis or Green Tea. Camellia sinensis is particularly rich in catechins and polyphenolic compounds. Catechin derivatives with 7-9 carbons have shown prominent antiviral activities. These catechin compounds have inhibitory actions on all six influenza virus subtypes, including various influenza viruses (A/H3N2, A/H1N1and B type), H9N2 and H2N2. These compounds vigorously block binding of the viruses on red blood cells [62]. The active components of Camellia sinensis are various polyphenols like epigallocatechin (EGC). epicatechin-3-gallate, epicatechin and epigallocatechin3-gallate (EGCG). Camellia sinensis catechins have distinct antimutagenic, anticarcinogenic, anti-inflammatory, antimicrobial and antioxidative properties which help to build strong immunity. Camellia synesis has viricidal properties due to its ability to inhibit flu virus replication [63].

\section{Conclusion}

COVID-19 pandemic has caused a worldwide threat, even though the current situation is relatively under control, the communities across the world are not well prepared to handle the outbreak from mutated or resistant strains of these viruses. Currently, there is no COVID-19 effective and safe vaccine. When available, the vaccine would be stockpiled across the world. After the launch of the vaccine, there will be a short supply. The emergence of mutant and virulent strains will pose additional difficult problems. Providing effective treatment to masses in a pandemic situation will be challenging for developing and underdeveloped countries.

Consideringthecurrentsituationalternativeandcomplementary medicine based on traditional herbs offers plenty of options to help patients. Medicinal herbs possess various biological activity like immune-boosting, anti-inflammatory, antiviral and antioxidative properties which can be successfully applied for managing current COVID-19 pandemic. Nutrition coupled with medicinal herbs provide a possibility to control viral infections. Herbal medicine, along with good nutrition, would be useful faster recovery. Many novel clinically tested herbal formulation is emerging, for example, Lianhuaqingwen (LH), a Chinese patent medicine composed of 13 herbs, offered alternative treatment for SARS-CoV-2 and other viral infections. Currently, many new polyherbal formulations are emerging, which are undergoing clinical trials for COVID-19 infections. Lianhuaqingwen inhibits Influenza viral propagation and regulates immune function and performs similar therapeutic effectiveness with Oseltamivir in reducing the H1N1 virus infection [64-66].

Every culture has a rich knowledge of traditional medicinal herbs which are being used since ages. There are several multiherb formulations which are used traditionally for the prevention and management of the respiratory viral disease. It's time to take a more in-depth look and investigate using scientific technology for the potential drug for emerging respiratory viruses. As like all traditional medicine, the various combination is readily available, which are safe and inexpensive. Traditional medicine can benefit a large population of the world, especially in developing and underdeveloped countries with limited or late access to vaccines and other therapies. Traditional medicine may also exhibit a generic efficacy against other viral infections with a similar pathological mechanism. The key challenges remain when we test the safety and efficacy of the combination of various drugs or traditional medicine the way we do it now is we often look at different mode of action and how certain drugs or traditional medicine works.

Can we combine these potential antiviral compounds to leverage for coordinated activity against these aggressive pathogens that we don't understand very well? The challenge is that it takes a long time and resources to know how to optimally design these potential antiviral candidate combinations to get maximum benefits. In the future, we can by leveraging different technologies like artificial intelligence and digital medicine; we can find optimal combinations much more quickly than by using traditional approaches. We may be talking about days to maybe just a couple of weeks. When that happens instead of trying many different repurposed combinations, we can arrive at an answer a lot faster and be able to help patients more efficiently. This review illustrates an overview of the potential herbs which can be combined to offer holistic solutions to assist in handling the current COVID-19 pandemic and also to cope with the next pandemic that might appear in the future.

\section{References}

1. Fung TS, DX Liu (2019) Human Coronavirus: Host-Pathogen Interaction. Annu Rev Microbiol 73: 529-557.

2. Du Toit A (2020) Outbreak of a novel coronavirus. Nat Rev Microbiol 18: 123. 
3. Carlos WG, Charles D, Bin Cao, Susan P (2020) Novel Wuhan (2019$\mathrm{nCoV}$ ) Coronavirus. Am J Respir Crit Care Med 201(4)

4. Zhou P, XL Yang, XG Wang, BEN H, L Zhang, et al. (2020) A pneumonia outbreak associated with a new coronavirus of probable bat origin. Nature 579: 270-273.

5. Mulangu S, Lori D, Ricard D, Oliver T, Michael P, et al. (2019) A Randomized, Controlled Trial of Ebola Virus Disease Therapeutics. N Engl J Med 381(24): 2293-2303.

6. Nkengasong J (2020) China's response to a novel coronavirus stands in stark contrast to the 2002 SARS outbreak response. Nat Med 26: 310311.

7. Huang C, Y Wang, Xinwang L, Lili Ren (2020) Clinical features of patients infected with 2019 novel coronavirus in Wuhan, China. Lancet 10233 497-506.

8. Wang D, B Hu, Chang Hu, Fang Zhu, Xing Liu, et al. (2020) Clinica Characteristics of 138 Hospitalized Patients With 2019 Novel Coronavirus-Infected Pneumonia in Wuhan, China. JAMA 323(11): 1061-1069.

9. Wu P, Xin H, Eric Y, Jessica Y, Kathy SM, et al. (2020) Real-time tentative assessment of the epidemiological characteristics of novel coronavirus infections in Wuhan, China, as at 22 January 2020. Euro Surveill 25(3).

10. Thevarajan I, Nguyen THO, Koutsakos M, Julian D, Leon C, et al. (2020) Breadth of concomitant immune responses prior to patient recovery: a case report of non-severe COVID-19. Nat Med 26: 453-455.

11. Verity R, Okell LC, Dorigatti I, Peter W, Charles W, et al. (2020) Estimates of the severity of coronavirus disease 2019: a model-based analysis. Lancet Infect Dis 20(6): 669-677.

12. Ruan S (2020) Likelihood of survival of coronavirus disease 2019. Lancet Infect Dis 20(6): 630-631.

13. Ren Jl, AH Zhang, XJ Wang (2020) Traditional Chinese Medicine for COVID-19 Treatment. Pharmacological Research 104743.

14. Iwu MW, Duncan AR, Okunji CO (1999) New antimicrobials of plant origin. In: Perspectives on New Crops and New Uses. Janick J (edn.) ASHS Press, VA, USA 457-462.

15. Gupta A (2003) Quality Standards of Indian Medicinal Plants (Volume 1). Indian Council of Medical Research, New Delhi, India.

16. (2003) Organization WH. Traditional medicine: World Health Organization Fact Sheet, No. 134, USA.

17. Kala CP, Dhyani PP, Sajwan BS (2006) Developing the medicinal plants sector in northern India: challenges and opportunities. J Ethnobiol Ethnomed 2(1): 32.

18. Kurokawa M, Shimizu T, Watanabe W, Shiraki K (2010) Development of new antiviral agents from natural products. Open Antimicrob Agents J 2 : 49-57.

19. Perera C, Efferth T (2012) Antiviral medicinal herbs and phytochemicals. J Pharmacognosy 3(1): 45-48.

20. Alves RR, Rosa IM (2007) Biodiversity, traditional medicine and public health: Where do they meet? J Ethnobiol Ethnomed 3: 14

21. Abdullahi AA (2011) Trends and challenges of traditional medicine in Africa. Afr. J. Tradit. Complement. Altern Med 8: 115-123.

22. Yakubo S, Ito M, Ueda Y, Okamoto H, Kimura Y (2014) Pattern classification in kampo medicine. Evid Based Complement Altern Med 2014.

23. Mogami S, Hattori T (2014) Beneficial effects of rikkunshito, a Japanese kampo medicine, on gastrointestinal dysfunction and anorexia in combination with Western drug: A systematic review. Evid Based Complement Altern Med 2014: 519035.

24. Lone AH, Ahmad T, Anwar M, Sofi G, Imam H, et al. (2012) Perception of health promotion in Unani herbal medicine. J Herb Med 2(1): 1-5.
25. Jabin F (2011) A guiding tool in Unani Tibb for maintenance and preservation of health: A review study. Afr J Tradit Complement Altern Med 8: 140-143.

26. Mishra L, Lakshmi C (2004) Scientific Basis for Ayurvedic Therapies, CIRC Press.

27. (2020) Ayush advisory from ministry of ayush for meeting the challenge arising out of spread of corona virus (covid-19) in India AYUSH 2020 India.

28. Warrier PK (1995) Indian 9 Medicinal Plants. Orient Longman, India.

29. Pompei R, Flore O, Marccialis M A Panni, B Laddo (1979) Glycyrrhizic acid inhibits virus growth and inactivates virus particles. Nature, 281(5733): 689-690.

30. Nose M, K Terawaki, K Oguri, Y Ogihara, Y Shimatsu, et al. (1998) Activation of macrophages by crude polysaccharide fractions obtained from shoots of Glycyrrhiza glabra and hairy roots of Glycyrrhiza uralensis in vitro. Biological and Pharmaceutical Bulletin 21(10): 11101112

31. Wagner H, Jurcic K (2002) Immunological studies of Revitonil, a phytopharmaceutical containing Echinacea purpurea and Glycyrrhiza glabra root extract. Phytomedicine 9(5): 390-397.

32. Cinatl J, Morgenstern B, Bauer G, Chandra P, Rabenau H, et al. (2003) Glycyrrhizin, an active component of liquorice roots, and replication of SARS-associated coronavirus. Lancet 361(9374): 2045-2046.

33. Orient H, Hansen BE, Willems M, Johannes T, Roman H, et al. (2006) Biochemical and histological effects of 26 weeks of glycyrrhizin treatment in chronic hepatitis C: a randomized phase II trial J Hepatol 45: 539-546.

34. Michaelis M, Geiler J, Naczk P, Sithisarn P, Ogbomo H, et al. (2010) glycyrrhizin inhibits highly pathogenic H5N1 Influenza A virus-induced pro-inflammatory cytokine and chemokine expression in human macrophages. Med Microbiol Immunol 199: 291-297.

35. Matsumoto Y, Matsuura T, Aoyagi H, Matsuda M, Hmwe, SS Date, et al. (2013) Antiviral activity of glycyrrhizin against hepatitis $\mathrm{C}$ virus in vitro. PloS one 8(7).

36. Domina Petric (2020) Glycyrrhizin and Coronaviruses, Projects: Antimicrobial agents COVID-19 preprints March 2020.

37. Chen H, Du Q (2020) Potential Natural Compounds for Preventing SARSCoV-2 (2019-nCoV) infection. Preprints v 2: 2020010358.

38. Kuhn M, Winston D (2007) Winston \& Kuhn's Herbal Therapy \&Supplements: A Scientific and Traditional Approach, Lippincott Williams \&Wilkins, Philadelphia, Pa, USA

39. Phadke SA, Kulkarni SD (1989) Screening of in vitro antibacterial activity of Terminalia chebula, Eclapta alba and Ocimum sanctum. Indian Journal ofMedical Sciences 43(5): 113-117.

40. Rai MK (1996) In vitro evaluation of medicinal plant extracts against Pestalotiopsis mangiferae," Hindustan Antibiotics Bulletin 38(1-4): 5356.

41. Kumar R, Singh DP, Chaturvedi VK, Pathak RC (1997) A note on antiviral property of neem (Melia azadirachata) and 0 sanctum (Ocimum sanctum) against Newcastle disease virus. Journal of Comparative Microbiology, Immunology and Infectious Diseases 18: 192.

42. Ghoke SS, Sood R, Kumar N, A K Pateriya, S Bhatia, et al. (2018) Evaluation of antiviral activity of Ocimum sanctum and Acacia arabica leaves extracts against H9N2 virus using embryonated chicken egg model. BMC Complement Altern Med 18(1): 174.

43. Castrillo JL, Carrasco L (1987) Action of 3-methylquercetin on poliovirus RNA replication. Journal of Virology 61(10): 3319-3321.

44. Vrijsen R, Everaert L, Hoof LMV, Vlietinck A J, Berghe D A, et al. (1987) Thepoliovirus-inducedshut-offofcellularproteinsynthesisper-sists in 
the presence of 3-methylquercetin, flavonoid which blocks viral protein and RNA synthesis. Antiviral Research 7(1): 35-42.

45. Zandi K, Teoh BT, Sam SS, Wong PF, Mustafa M, et al. (2011) Antiviral activity of four types of bioflavonoid against dengue virus type-2. Virology Journal 8(1): 3586.

46. Takimoto T, Taylor GL, Connaris HC, Crennell SJ, Portner A (2002) Role of the Hemagglutinin-Neuraminidase Protein in the Mechanism of Paramyxovirus-Cell Membrane Fusion. Journal of Virology 76(24): 13028-13033.

47. Wu L, Chen Y, Gao H, Yin J, Huang L (2016) Cadmium-induced cell killing in Sacharomyces cerevisiae involves increases in intracellular NO levels. FEMS Microbiol Lett 363(6).

48. Yao C, Xi C, Hu K, Gao W, Cai X, et al. (2018) Inhibition of entero virus71 replication and Viral 3C protease by quercetin. Virology Journal 15(1): 116-116.

49. Qiu X, Kroeker A, He S, Kozak R, Audet J, et al. (2016) Prophylactic Efficacy of Quercetin3- -O-d-Glucoside against Ebola Virus Infection. Antimicrobial Agents and Chemotherapy 60(9): 5182-5188.

50. Duke W (2009) Medicine: Cocos nucifera Folk Medicine 2009.

51. Kabara JJ (1978) The Pharmacological Effect of Lipids, American Oil Chemist's Society, Champaign, Ill, USA.

52. Projan SJ, Skrobot SK, Novick RP (1996) Glycerol monolaurate inhibits the production of beta-lactamase, toxic shock toxin1, and other staphylococcal exoproteins by interfering with signal transduction. Journal of Bacteriology 176: 4204-4209.

53. Ganguli S (1994) Neem: a therapeutic for all seasons. Current Science 82(11): 1304.

54. Parida MM, Upadhyay C, Pandya G, AM Jana (2002) Inhibitory potential of neem (Azadirachta indica Juss) leaves on dengue virus type-2 replication. Journal of Ethnopharmacology 79(2): 273-278.

55. Badam L, Joshi SP, Bedekar SS (1999) In vitro antiviral activity of neem (Azadirachta indica A Juss) leaf extract against group B Coxsackieviruses. Journal of Communicable Diseases 31(2): 79-90.

ISSN: $2574-1241$

DOI: 10.26717/BJSTR.2020.28.004643

Shabbir Moochhala. Biomed J Sci \& Tech Res

This work is licensed under Creative

Commons Attribution 4.0 License

Submission Link: https://biomedres.us/submit-manuscript.php
56. Gu W, Zhang Y, Hao XJ, Yang FM, Sun QY (2014) Indole alkaloid glycosides from the aerial parts of Strobilanthes cusia. J Nat Prod 77: 2590-2604.

57. Zhou B, Yang Z, Feng Q Liang X, Li J, et al. (2017) Aurantiamide acetate from baphicacanthus cusia root exhibits anti-inflammatory and antiviral effects via inhibition of the NF-kappaB signaling pathway in Influenza A virus-infected cells. J Ethnopharmacol 199: 60-67.

58. Tsai YC, Lee CL, Yen HR, Chang YS, Lin YP (2020) Antiviral action of Tryptanthrin isolated from Strobilanthes cusia leaf against human coronavirus NL63. Biomolecules 10: 366.

59. Gu W, Wang W, Li XN, Zhang Y, Wang LP, et al. (2015) A novel isocoumarin with anti-influenza virus activity from Strobilanthes cusia. Fitoterapia 107: 60-62.

60. Liau BC, Jong TT, Lee MR, Chen SS (2007) LC-APCI-MS method for detection and analysis of tryptanthrin, indigo, and indirubin in daqingye and banlangen. J Pharm Biomed Anal 43: 346-351.

61. Lai JL, Liu YH, Liu C, Qi MP, Liu RN, et al. (2017) Indirubin Inhibits LPSInduced Inflammation via TLR4 Abrogation Mediated by the NF-kB and MAPK Signaling Pathways. Inflammation 40: 1-12.

62. Li Y, But PPH, Vincet EC (2005) Antiviral activity and mode of action of caffeoylquinic acids from Schefflera heptaphylla (L) Frodin. Antiviral Research 68(1): 1-9.

63. Song JM, Park KD, Lee LH, Byun YH, Park JH, et al. (2007) Biological evaluation of anti-influenza viral activity of semi-synthetic catechin derivatives. Antiviral Research 76(2): 178-185.

64. Ding Y, L Zeng, R Li, Q Chen, B Shou, et al. (2017) The Chinese prescription lianhuaqingwen capsule exerts anti-influenza activity through the inhibition of viral propagation and impacts immune function. BMC Complement Altern Med 17(1): 130.

65. Lu H (2020) Drug treatment options for the 2019-new coronavirus (2019-nCoV). Biosci Trends 14(1): 69-71.

66. Duan ZP, Jia ZH, Jian Z, S Liu, Y Chen, et al. (2011) Natural herbal medicine Lianhuaqingwen capsule anti-influenza A (H1N1) trial: a randomized, double blind, positive controlled clinical trial. Chin Med J (Engl) 124(18): 2925-2933.

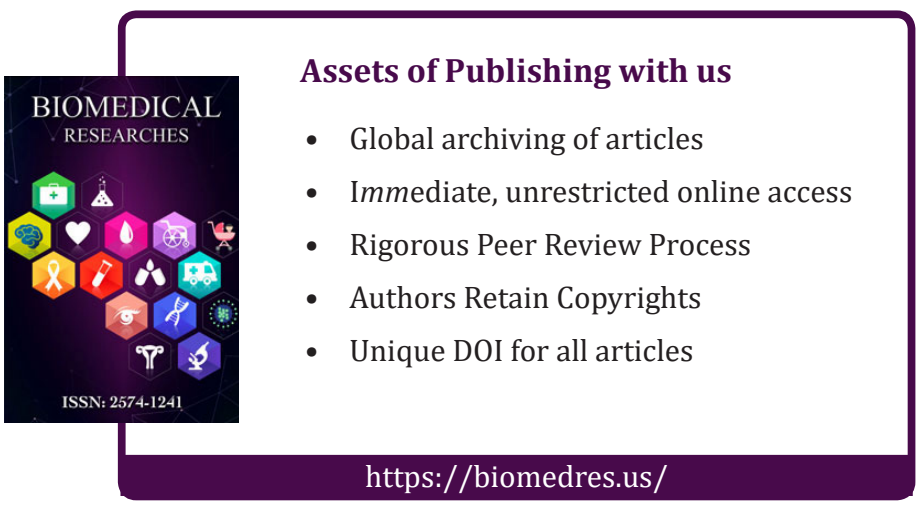

Copyright@ Shabbir Moochhala | Biomed J Sci \& Tech Res | BJSTR. MS.ID.004643. 